\title{
SiPM characterisation for cosmic muon veto detector of mini-ICAL
}

\author{
Mamta Jangra, ${ }^{a, b, *}$ Gobinda Majumder, ${ }^{b}$ Mandar N Saraf ${ }^{b}$ and B. Satyanarayana ${ }^{b}$ \\ ${ }^{a}$ Homi Bhabha National Institute, Mumbai \\ ${ }^{b}$ Tata Institute of Fundamental Research, Mumbai \\ E-mail: mamta.jangra@tifr.res.in
}

The prototype detector of Iron CALorimeter (ICAL) experiment at the India-based Neutrino Observatory, mini-ICAL is currently running at IICHEP Madurai, India. An active cosmic muon veto (CMV) detector is going to house the mini-ICAL from top and sides except the front side. CMV consists of $5 \mathrm{~cm}$ wide extruded plastic scintillators with embedded two WLS fibers to propagate scintillation light and SiPM at both ends of fibres as photosensors for detecting photons. The SiPM will be calibrated using an LED ultrafast driver. A small experimental setup is built to characterise the SiPM along with an extruded scintillator strip to optimise the operating over voltage, threshold of SiPM signals and the veto criteria by observing muon signals and the noise rates in the SiPM. These optimisations along with other characteristics of SiPM, e.g., cross-talk, after pulse, recovery time etc are discussed in this paper.

*** The European Physical Society Conference on High Energy Physics (EPS-HEP2021), ***

** 26-30 July 2021 ***

** Online conference, jointly organized by Universität Hamburg and the research center DESY ***

${ }^{*}$ Speaker 


\section{Introduction}

The cosmic ray muon flux on the earth's surface constitutes a huge background for experiments looking for neutrino interactions. An experiment setup at a depth of $\sim 1 \mathrm{~km}$ will reduce cosmic muon background by a factor of $10^{6}$ and a shallow depth of $\sim 100 \mathrm{~m}$ will give a rejection factor of $10^{2}$. To achieve a rejection factor of $10^{6}$ at depth of $100 \mathrm{~m}$, an active Cosmic Muon Veto (CMV) detector is required with veto efficiency $>99.99 \%$. The mini-ICAL, a prototype of Iron CALorimeter, consists of an 85-ton magnet built using 10 layers of $2 m \times 2 m$ glass Resistive Plate Chambers (RPCs) and 11 layers of $5.6 \mathrm{~cm}$ thick soft iron plates and is operational at Madurai, India. A CMV detector is being built on top of mini-ICAL with extruded plastic scintillators with embedded WLS fibres to propagate light and SiPM to detect photons.

\section{Experimental setup}

The experimental setup requires a $(60 \mathrm{~cm} \times 5 \mathrm{~cm} \times 2 \mathrm{~cm})$ extruded scintillator strip with embedded WLS fibres into the holes, an acrylic fibre guide bar to mount the Counter MotherBoard (CMB), a total of 4 SiPMs to readout on both sides as shown in Fig. 1. SiPMs are powered using a Keithley sourcemeter (Model 2400) with a common bias.

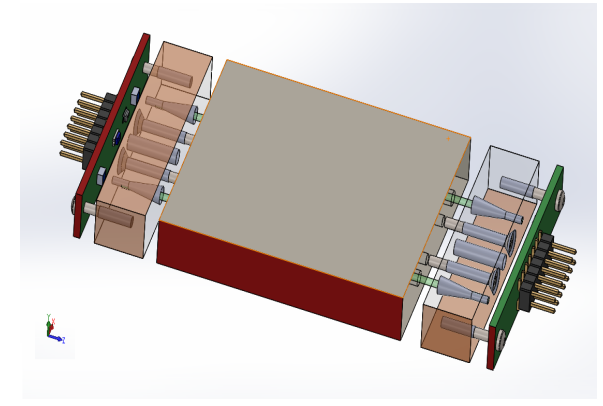

Figure 1: Scintillator counter schematic.

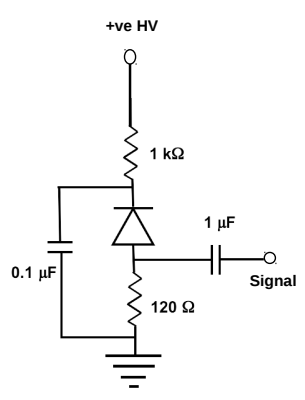

Figure 2: SiPM circuit diagram.

\section{LED calibration}

An ultrafast LED driver (CAEN SP5601) is used for characterising SiPM. The integrated charge is calculated using the equation :

$$
Q_{t o t}=\frac{1}{R} \int_{t_{0}}^{t_{1}} V(t) d t
$$

where $\mathrm{R}=120 \Omega$ as shown in Fig. 2. Total collected charge is fitted with a function:

$$
f(q)=\operatorname{Landau}(q)+\sum_{n=0}^{N-1} R_{n} \times \exp \left(-\frac{(q-n \cdot \mu)^{2}}{2 \sigma_{n}^{2}}\right)
$$

where $\mathrm{N}$ is the number of photoelectron (p.e.) peaks, $R_{n}$ is peak height of $n^{\text {th }}$ p.e., $\mu$ is the gain of SiPM and $\sigma_{n}$ is the gaussian width of $n^{\text {th }}$ p.e. peak, which has small dependency on $n$. Fitted spectrums are shown in Fig. 3. The estimated gain, which is the average average gap between consecutive peaks from the fit which is shown in Fig. 4(a) as a function of bias voltage. Gain is linear above a threshold voltage, but the dark current is increased exponentially, as shown in Fig. 4(b). 

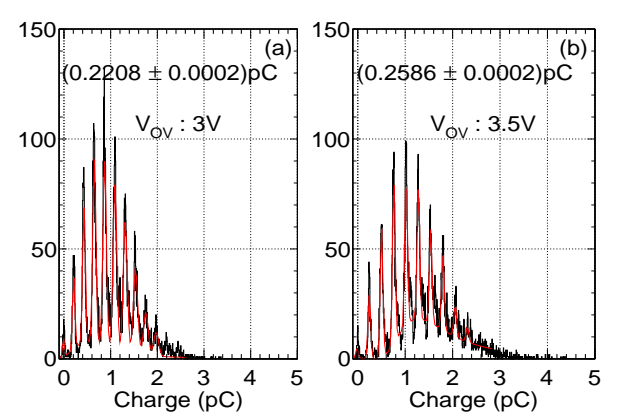

Figure 3: Photoelectron peaks at $V_{o v}=3 V$.
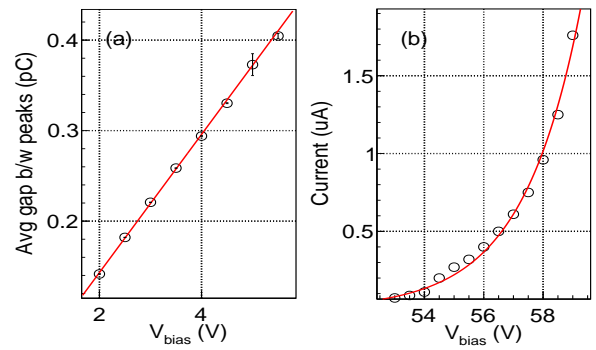

Figure 4: (a) Single p.e. charge value, (b) dark current drawn by SiPM w.r.t. bias voltage.

\section{Calibration using noise data and radioactive source data}

For noise data as well as radio-active source data, a random trigger is generated using one of the SiPM channels and data from other SiPMs mounted on the extruded scintillator is collected. In case of radio-active source data, ${ }^{22} \mathrm{Na}$ is placed on top of the extruded scintillator. Fig. 5 and Fig. 6 shows the total charge collected from noise data and radioactive source data respectively. Gain is calculated by noting the average gap between the consecutive peaks. Results of all three calibration techniques are consistent with each other.

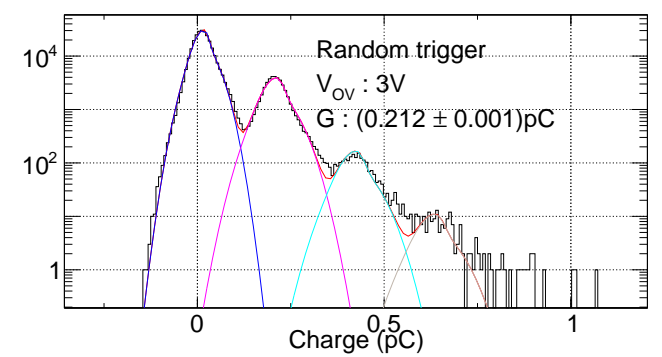

Figure 5: Photoelectron peaks at $V_{o v}=$ $3 V$ from noise data.

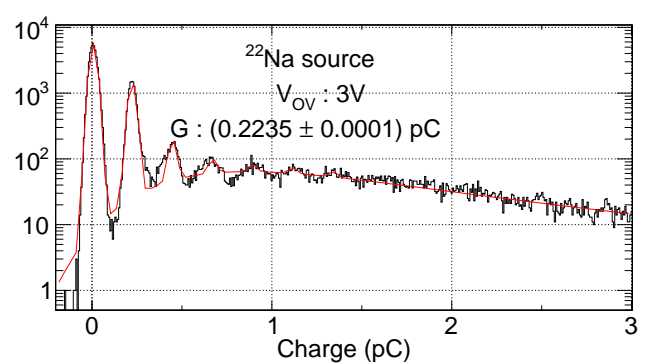

Figure 6: Photoelectron peaks at $V_{o v}=$ $3 V$ from radio-active source data.

\section{Correlated noise}

Amplified output waveforms from SiPM are stored using random triggers in a dark room for measuring correlated noise i.e. afterpulse and crosstalk rates[1]. The time difference between consecutive pulses and amplitude of the second pulse is used for the measurement as shown in Fig. 7 to estimate the delayed cross-talk, after-pulse and prompt cross talk.

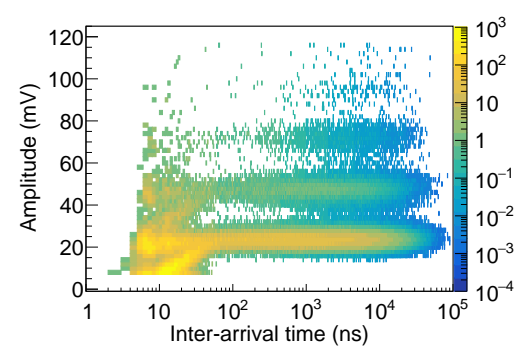

Figure 7: Amplitude versus time difference distribution at $V_{o v}=3 \mathrm{~V}$.

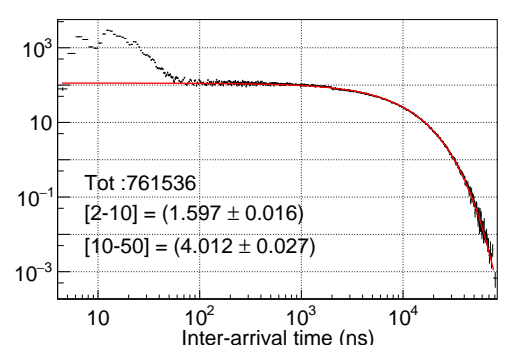

Figure 8: Correlated noise in log-log scale at $V_{o v}=3 \mathrm{~V}$. 
The estimation of delayed cross-talk[2-10], after-pulse[10-50] are shown in Fig.8.

\section{Recovery time of SiPM}

Recovery time is the time taken by SiPM to regain it's former state after an avalanche. From the afterpulse slope as shown in Fig. 7, mean amplitude is measured for corresponding time values as shown in Fig.9. The four peaks in Fig.9(a) are coming from after pulse, delayed cross talk, after pulse with delayed cross talk and delayed cross talk with subsequent prompt cross talk. The distribution Fig.9(a) is fitted with individual four Gaussian functions and the distribution Fig.9(b) is fitted with :

$$
f(\Delta t)=p_{0} \times\left[1-\exp \left(-\frac{\left(\Delta t-t_{0}\right)}{\lambda}\right)\right]
$$

where $\lambda$ is the decay constant (recovery time) which is found to be $(22.57 \pm 0.34) \mathrm{ns}$ at $V_{o v}=4 \mathrm{~V}$.

\section{Optimisation of signal efficiency vs noise rate}

Noise data collection is done using random triggers. For cosmic data, coincidence technique is used to ensure that the muon has passed through the extruded scintillator strip. Cosmic muon efficiency as well as noise rate of SiPM is calculated at different values of $V_{o v}$ and at different charge thresholds as shown in Fig.10(a) and Fig.10(b) respectively. Comparing these two, it is decided to operate SiPMs at an overvoltage of (2-3) V for cosmic data.
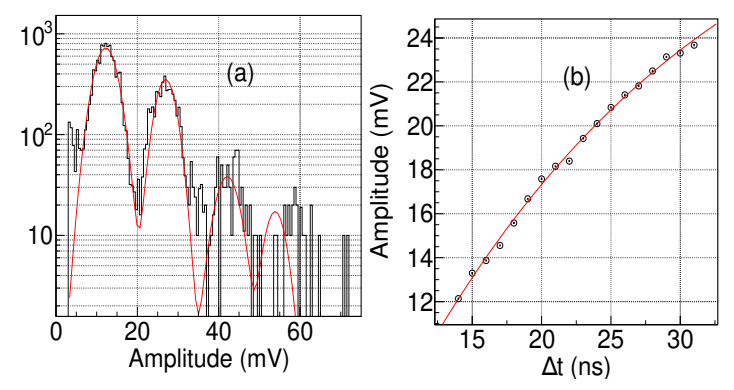

Figure 9: (a) Amplitude of the second pulse for $\Delta t=15-16 \mathrm{~ns}$ and (b) Mean amplitude of after pulse as a function of $\Delta t$ at $V_{o v}=4 \mathrm{~V}$.
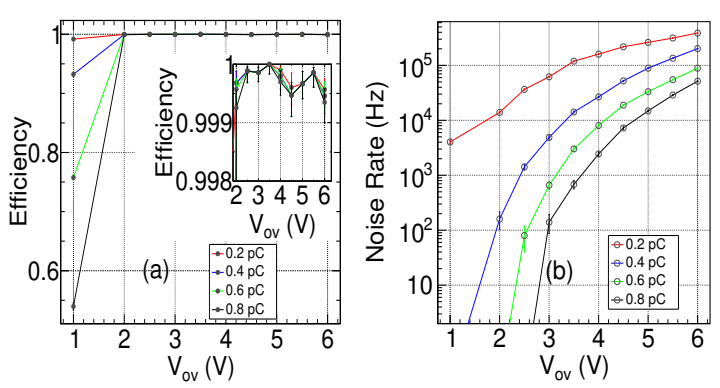

Figure 10: (a) Cosmic muon efficiency and (b) Noise rate of SiPM as a function of $V_{o v}$.

\section{Conclusion}

Optimal value of $V_{o v}$ is decided to be $(2-3) \mathrm{V}$ by studying noise rate of SiPM and cosmic muon detection efficiency at different $V_{o v}$. Correlated noise is also studied to see it's effect on various parameters and it is found to be $\sim 10 \%$ of the total noise at $V_{o v}=3 \mathrm{~V}$.

\section{Acknowledgements}

We would like to sincerely thank HBNI and all the members of INO collaboration.

\section{References}

[1] Robert Klanner 2019 Nuclear Inst. and Methods in Physics Research. A 926 36-56. 